\title{
Strategies and Factors Associated With Top Performance in Primary Care for Diabetes: Insights From a Mixed Methods Study
}

Leif I. Solberg, $M D^{1}$

Kevin A. Peterson, MD, MPH ${ }^{2}$

Helen Fu, RN, $\mathrm{PbD}^{2}$

Milton Eder, $P b D^{2}$

Racbel Jacobsen, MPH, RD

Caroline S. Carlin, $P b D^{2}$

'HealthPartners Institute, Minneapolis Minnesota

${ }^{2}$ University of Minnesota, Minneapolis, Minnesota
Conflicts of interest: authors report none.

\section{CORRESPONDING AUTHOR}

Leif I. Solberg

HealthPartners Institute

PO Box 1524, MS\#21112R

Minneapolis, MN 55440-1524

leif.i.solberg@healthpartners.com

\begin{abstract}
PURPOSE The aim of this study was to determine what strategies and factors are most important for high performance in the primary care of patients with diabetes.

METHODS We performed a mixed-methods, cross-sectional, observational analysis of interviews and characteristics of primary care clinics in Minnesota and bordering areas. We compared strategies, facilitators, and barriers identified by 31 leaders of 17 clinics in high-, middle-, and low-performance quartiles on a standardized composite measure of diabetes outcomes for 416 of 586 primary care clinics. Semistructured interview data were combined with quantitative data regarding clinic performance and a survey of the presence of care management processes.
\end{abstract}

RESULTS The interview analysis identified 10 themes providing unique insights into the factors and strategies characterizing the 3 performance groups. The main difference was the degree to which top-performing clinics used patient data to guide proactive and outreach methods to intensify treatment and monitor effect. Top clinics also appeared to view visit-based care management processes as necessary but insufficient, whereas all respondents regarded being part of a large system as mostly helpful.

CONCLUSIONS Top-performing clinic approaches to diabetes care differ from lower-performing clinics primarily by emphasizing data-driven proactive outreach to patients to intensify treatment. Although confirmatory studies are needed, clinical leaders should consider the value of this paradigm shift in approach to care.

Ann Fam Med 2021;19:110-116. https://doi.org/10.1370/afm.2646.

\section{INTRODUCTION}

$\mathrm{I}$ $\mathrm{n}$ a recent study of US National Health and Nutrition Examination Survey data, Kazemian et al reported that measures of diabetes care outcomes did not improve from 2005 to $2016 .^{1}$ This was true despite significant increases in the proportion of patients with college education and health insurance coverage over those years and despite improvements in treatment and several large national campaigns to improve diabetes control and decrease cardiovascular risk factors. ${ }^{2-4}$ A similar lack of improvement over this period was reported by Tummalapalli et al. ${ }^{5}$

Kazemian et $\mathrm{al}^{1}$ pointed out the example of Minnesota, where the percentage of patients with diabetes who achieved optimal diabetes care measures increased from $12 \%$ to $45 \%$ during the period 2004 to 2017, in contrast to national sample results plateauing at 23\% from 2013 to 2016. They attributed this difference to an emphasis on performance monitoring and public reporting.

As part of a study of care management processes associated with the best outcomes among Minnesota primary care clinics, we conducted a mixed-methods study to determine what factors distinguish high-performing clinics from those doing less well. We had access to standardized quantitative performance and care process data from a majority of clinics 
in the state, along with qualitative interviews with leaders of selected clinics. The quantitative data allowed us to identify the best clinics for interviews and to compare their context and degree of systematization. Interviewees reported strategies and factors they believed contributed most to their performance on standardized composite measures for diabetes control.

\section{METHODS}

The Understanding Infrastructure Transformation Effects on Diabetes study recruited 451 (77\%) of the 586 primary care practices that submitted standardized data to Minnesota Community Measurement (MNCM), a nonprofit organization that has collected and publicly reported standardized medical performance measures since 2006. State law has required clinics to submit diabetes performance data since 2011. ${ }^{6}$ Quantitative analyses of this data set have been published. ${ }^{7,8}$

\section{Quantitative Data}

Study participation depended on having a clinic leader complete a 112-question survey in 2017 regarding the presence of various care management processes to support high-quality care for patients with chronic medical conditions. The survey was created and tested for reliability by the National Committee for Quality Assurance and has been widely used. ${ }^{9-11}$ We received completed surveys from 416 clinics (92\% of participating clinics). Respondents reported whether each care management process was present, and the score was based on the percentage of eligible processes present. We identified questions that specifically addressed care management processes for diabetes so we could analyze an overall score and a subscore specific to diabetes. Example questions included the following:

- Does your clinic use checklists of tests or interventions that are needed for prevention or monitoring of chronic illness?

- Does your clinic have nonphysician staff who are specially trained and designated to educate patients in managing their chronic illness?

- Does your clinic provide guideline-based reminders for services a diabetes patient should receive that appear when seeing the patient?

- Does your clinic provide or refer patients to formal support programs to assist in self-management for chronic conditions?

Respondents were also asked to rate their clinic's priority for improving diabetes care in the next year on a scale of 0 (not a priority) to 10 (highest priority). Another key quantitative data set for the present study was obtained from MNCM for performance data collected in 2016 and reported in 2017. For diabetes, these measures included the percentage of patients with diabetes at each clinic with controlled diabetes (glycated hemoglobin $<8 \%$ ), controlled hypertension (blood pressure $<140 / 90 \mathrm{~mm} \mathrm{Hg}$ ), controlled hyperlipidemia (lowdensity lipoprotein cholesterol $<100 \mathrm{mg} / \mathrm{dL}$ or taking a statin), taking aspirin unless contraindicated, and who were nonsmokers. We used an MNCM composite (all or none) measure counting each patient with diabetes controlled or not controlled depending on whether they met all 5 measures.

The MNCM data did not include information on the race/ethnicity, wealth, income, or educational status of each clinic's patients. Therefore, we matched patient addresses to 2015 five-year average American Community Survey data to identify patient characteristics by zip code. ${ }^{12}$ Each characteristic was averaged across patients within a clinic, and clinics were ranked by quartile for each population factor. Clinics were also identified as being located in the Twin Cities metropolitan area or in other parts of the state.

\section{Clinic Selection}

To identify clinics for interviews, we matched pairs of clinics with high and low scores on the MNCM composite performance measure but similar average propensity scores, as calculated by a patient-level regression that predicted achievement of the composite measure adjusted for age, sex, presence of vascular disease or depression, presence of type 1 diabetes, insurance type, and average wealth, income/education, and race/ ethnicity in the patient's zip code, allowing us to identify clinics performing better or worse than might have been predicted by the characteristics of their patient population. Of 9 metropolitan and 19 nonmetropolitan pairs of clinics with similar expected performance on the diabetes measure but actual performance at least 2 quartiles apart, we selected 10 pairs of clinics with balanced metropolitan/nonmetropolitan locations and from varied types of medical groups.

We invited leaders of clinics that completed the earlier survey on care management processes to participate in a 20 - to 30 -minute interview regarding their approach to diabetes care. Three clinics $(2$ identified as performing lower than expected) declined participation, and we were unable to identify equivalent replacements, resulting in interviews at 17 clinics (Table 1), 9 from the metropolitan area and 8 from nonmetropolitan areas. Typical of Minnesota primary care clinics, 15 were part of 8 large medical groups (1162 clinics), 1 was a solo clinic, and 1 was in a 3 -clinic organization. Of the 31 leaders interviewed, there were 5 physicians, 3 nurse practitioners, 10 clinic managers, and 13 clinical supervisors. Clinicians and nonclinicians were usually both present. 


\section{Qualitative Data Collection}

Interviews were performed by 1 of 3 experienced coauthors (L.S. and K.P. physicians, M.E. anthropologist) following a semistructured interview guide with open-ended questions and probes regarding the following:

- The factors that contributed most to their MNCM diabetes scores

- The strategies that were most successful in improving care for patients with diabetes

- The most important barriers and facilitators to improving diabetes care

- The most important help that they received or would like to receive from their organization

- Their awareness of comparative performance scores from MNCM

After obtaining consent, we conducted 20 - to

30-minute interviews at the clinic site with all participants simultaneously, with recording to assure accuracy and completeness. All interview data were transcribed verbatim by a professional service that removed personnel identifiers and replaced clinic names with codes. We used NVivo version 12 (QSR International) for data management.

\section{Analysis}

The analysis team consisted of 5 coauthors, each of whom reviewed the interviews independently and then met 2 to 4 times per month over a period of 8 months to achieve consensus. We used grounded theory, with the initial coding framework constructed as a directed content analysis from the domains and questions used in the interviews-strategies, facilitators, and barriers. ${ }^{13}$ The framework and individual codes were then modified by consensus as we went through the interviews together, using a combination of individual reviews followed by group discussion to clarify and standardize codes across all interviews. This was followed by agreeing on observations and then conducting a summative analysis for themes, combining frequency counts with observations in a constant comparative approach. ${ }^{14}$ These steps were undertaken for high- and low-performance groups and comparing them. We used a similar approach for the middle-quartile clinics to test the themes developed from the original high/low comparison. Differences of opinion were discussed until consensus was achieved. A detailed codebook with definitions, a quantitative summary of clinic comments for each code, and an audit trail ensured rigorous analysis, but we did not check results with interviewees. ${ }^{15}$ The study was approved by the University of Minnesota Institutional Review Board.

\section{RESULTS}

Table 1 shows a quantitative comparison of the 3 interviewed clinic groups (high, middle, low). There was little difference in score by metropolitan/nonmetropolitan location. Mean priority scores for improving diabetes care were associated with performance scores. The middle group had the greatest number of care management processes, both for diabetes and overall, and was also most likely to relate the importance of those processes (Table 2). Interestingly, the 2 clinics in small organizations were in the bottom (low) quartile, although other small groups performed well (see theme 6 below regarding clinic relationships to larger systems).

Whereas the original coding framework for interview data grouped all care strategies together, it soon became apparent that there were 2 different sets of strategies (Table 2): (1) care management processes to provide systematic delivery of services focused on a traditional visit-based relationship with patients, with individual patient responsibility for attendance and adherence to care plans. Services were limited to those patients who maintained engagement with the clinic. The content of the clinic survey was largely limited to supportive care management processes within this type of patient-clinic relationship ${ }_{i}(2)$ care management processes based on the clinic assuming a more proactive responsibility for ensuring that patients achieved targeted goals. Clinics using this approach started with regular reports that identified the degree to which each patient with diabetes met measurement goals so that clinic staff could use various outreach methods (mail, e-mail, telephone) to suggest visits, tests, or 


\begin{tabular}{ccccc}
\hline $\begin{array}{c}\text { Patients } \\
\text { on Medical } \\
\begin{array}{c}\text { Assistance, } \\
\text { Mean \% (SD) }\end{array}\end{array}$ & $\begin{array}{c}\text { MNCM } \\
\text { Composite } \\
\text { Score, } \\
\text { Mean \% (SD) }\end{array}$ & $\begin{array}{c}\text { Priority for } \\
\text { Improving } \\
\text { Diabetes Care, } \\
\text { Mean (SD) }\end{array}$ & $\begin{array}{c}\text { CMPs for } \\
\text { Diabetes, } \\
\text { Mean (SD) }\end{array}$ & $\begin{array}{c}\text { CMPs } \\
\text { Mean (SD) }\end{array}$ \\
\hline $13.0(7-19)$ & $54.8(51.3-61.1)$ & $9.2(7-10)$ & $66(33-93)$ & $62(27-95)$ \\
\hline $10.0(6-14)$ & $45.6(41.0-48.4)$ & $8.5(7-10)$ & $72(50-89)$ & $68(48-93)$ \\
\hline $15.2(9-19)$ & $36.3(33.3-39.1)$ & $8.0(7-10)$ & $54(43-64)$ & 61 (37-57) \\
\hline $12.3(6-19)$ & $48.3(33.3-61.1)$ & $8.4(7-10)$ & $68(11-98)$ & 65 (10-99) \\
\hline $13.9(0-55)$ & $44.8(17.6-63.7)$ & $8.4(0-10)$ & & \\
\hline
\end{tabular}

with timely and accurate reports that identified individual patients who were not at goal, so that they could address needs without waiting for patient visits or requests. The number of comments per code (Table 2) suggested little difference, but the content of the comments was dramatically different depending on the clinic's performance level. Below are comments from high-performing clinics:

"So, we get these patient opportunity lists every single month. And providers review them, staff review them, and then they do follow-up phone calls with patients- the patients maybe who haven't been in to have their $\mathrm{A}_{\mathrm{lc}}$ done." (Clinic $\mathrm{F}$ High)

treatment changes. Such clinics also used that information to take advantage of visits for any reason to reinforce those suggestions, providing what we called opportunistic care.

The above differentiation proved central for explaining the emerging differences among clinics in both performance and strategies. The methodic qualitative analytic process we used, combined with quantitative data on the frequency and distribution of codes by clinic (Table 2) and the use of iterative periods of reflection and discussion, allowed us to identify the following major themes:

1. Proactive care is a critical performance difference. The principal difference in approach to diabetes care among interviewed clinics was that high-performing clinics were much more likely to report using proactive care, whereas low-performing clinics said nothing about such strategies or identified barriers to their use Middle-performing clinics were also middling in their use of these strategies, and their comments described just starting this approach or seeing it as a wish. Highperforming clinics relied heavily on working actively,
"They also have a culture here of every patient, every time. So every time a patient is seen, we want to make sure all of their immunizations, their forms, their labs, everything is up to date." (Clinic O High)

"If patients don't come in, we have nurses looking at the lists. Every month, the provider gets the list and will go through who they want called." (Clinic H High)

2. Visit-based care management processes are necessary but insufficient for highest performance. We were surprised by how few comments there were across all performance groups regarding the care management processes that have been shown to be effective in the Chronic Care Model (reminders, self-management support, tracking systems, flowsheets, checklists, etc), ${ }^{17}$ with the exception of diabetes education. Within that context, the middle-performing group had the most comments regarding these processes and the highest scores on our survey of processes for diabetes (Table 1). This suggests that a clinic can obtain good performance by implementing these processes, but to break through to high performance, a paradigm shift

Table 2. Code and Comment Frequency

\begin{tabular}{lcccccc}
\hline & \multicolumn{3}{c}{ All Clinics } & & Codes per Clinic, No. \\
\cline { 2 - 7 } Domain & $\begin{array}{c}\text { Codes, } \\
\text { No. }\end{array}$ & $\begin{array}{c}\text { Comments, } \\
\text { No. }\end{array}$ & $\begin{array}{c}\text { Comments per } \\
\text { Code, No. }\end{array}$ & $\begin{array}{c}\text { High } \\
(\mathbf{n}=\mathbf{8})\end{array}$ & $\begin{array}{c}\text { Middle } \\
(\mathbf{n}=6)\end{array}$ & $\begin{array}{c}\text { Low } \\
(\mathbf{n}=3)\end{array}$ \\
\hline Traditional care management process strategies & 7 & 41 & 5.9 & 1.9 & 3.3 & 2.0 \\
Proactive outreach strategies & 4 & 46 & 11.5 & 3.0 & 2.7 & 2.0 \\
Facilitating factors - clinic & 14 & 86 & 6.1 & 5.8 & 5.2 & 3.0 \\
Facilitating factors - organizational & 7 & 64 & 9.1 & 4.2 & 3.0 & 4.0 \\
Barriers - clinic & 11 & 50 & 4.5 & 2.2 & 3.0 & 4.7 \\
Barriers - patient & 4 & 29 & 7.3 & 1.8 & 2.0 & 1.0 \\
Total & 47 & 316 & $\ldots$ & $\ldots$ & $\ldots$ & $\ldots$ \\
\hline
\end{tabular}


is needed to add proactive care. Below are comments from middle- and low-performing clinics:

"We print the after-visit summary and give them to the patients. We find a decent number of them lying either in the exam room where the patient was or in the lobby. What happens when they walk out the door is always a bit of a mystery." (Clinic Q Middle)

"I think just standardizing that previsit planning, I think that's the number one key for us. We have a lot of people that do it, but...there's some providers that don't want their staff to do it." (Clinic I Low)

3. In addition to care delivery strategies, facilitating factors must also be present. High-performing clinics were much more aware of facilitating factors, such as good patient access, clinician continuity, culture, leadership, and clinician and team engagement. Lowperforming clinics identified few of these facilitators, and the middle group was in between:

"We keep $25 \%$ of our primary care visits available for sameday use." (Clinic E High)

"Each patient's different, so we really approach each patient differently." (Clinic M High)

"We are continuously focusing on all quality measures, but we're highlighting a specific measure every month to make sure that everybody knows, again, what's required, what their part is." (Clinic F High)

4. Expanded care team roles are accepted. Clinic leaders at all performance levels talked about the importance of a variety of roles (eg, care coordinators, pharmacists) on the care team beyond the traditional clinician and assistant. Again, low-performing clinic leaders were more likely to describe additional roles as a wish rather than a reality:

"In 2017, we had a pharmacist in the clinic with us. Some of the clinics do, but we do not any longer. That was a helpful resource too." (Clinic D Low)

"And XXXX as a system also has care coordinators. We have diabetes educators, and we have medication therapy management by pharmacists, so all of those help contribute to our quality." (Clinic O High)

"We really have our MAs working at...close to the top of their scope. So I think that's a big part of the success that we have had." (Clinic E High)

\section{Community resource use and action on social} determinants were not described much. Only 2 leaders at any performance level discussed community resources for their patients with diabetes, and none described screening or action on patient social needs. Nearly all, however, talked about the care problems created by patient social limitations, especially financial barriers:
"They might not be able to see the dietitian because they don't have transportation. They might not be able to fill their medicines because they're too expensive." (Clinic F Low)

"Cost [is always] a barrier, 'I have to pay $\$ 3,000$ deductible and I don't want to come see you every 3 months because I have to pay for those visits.' " (Clinic M High)

6. Being part of a large system is regarded as mostly helpful. Most interviewed leaders viewed their larger organization as providing important information (especially accurate and timely patient-level goal-attainment reports), support services (eg, information technology), and opportunities to share learnings with other clinics:

"If we think we're doing everything correct and we still can't figure out why we're not meeting something, we can call in these specialists to come in to help us do a deep dive into the data, to look at our work flows." (Clinic F High)

"The other clinics and the executive leadership team. So they're on board, and they're helping just support the work that we do in the clinic." (Clinic F High)

"Every month...the report comes to us as a system, which is boiled down to a region, to a clinic, to the provider. We then take that report and take the data and filter it into our own site reports and share that with our teams on a monthly basis." (Clinic B Middle)

7. It helps to not have clinic/organizational barriers. High-performing clinic leaders had relatively little to say about barriers to care, and the ones mentioned were mild, whereas those of low- and middle-performing clinics frequently expressed concerns regarding clinician or staff turnover, time limitations, and individualistic clinician priorities:

"...there's...some providers that don't want their staff to do it [previsit planning]." (Clinic I Low)

"We are short probably 1 or 2 physicians right now within our group, so patients will say, I need to make an appointment, and they won't be able to get in for over a month." (Clinic R Low)

8. Clinic performance awareness matters. Leaders of high-performing clinics had greater awareness of their clinic's performance on quality measures than those of low- or middle-performing clinics, and the latter more often described confusion about measures. Clinic leaders were more concerned about their performance relative to other clinics in their medical group than to statewide rates. None of the clinic leaders described reporting comparative performance rates for individual clinicians:

"We also have a quality team which meets, and we focus on our quality scores. And each of us has a part in it and a role in that, helping with those scores and really digging into the whys - why we might have a low score or a high score in an area." (Clinic O High) 
9. There was little blaming of patients for deficiencies in performance rates. Although all leaders recognized that many of their patients had other higher priorities than diabetes treatment, it was rare for them to blame patient attitudes for clinic performance standings:

"If someone can't afford their insulin, it's a huge barrier...if we have patients that maybe go in and out of having coverage for visits, medications, and things like that, it's a big factor in our clinic." (Clinic D Low)

10. Establishing trust and good relationships between clinicians and patients is essential. When asked about what they would advise other clinics to do to improve scores, most leaders of high- or middle-performing clinics said that good, trusting patient relationships were critical for getting patient adherence to treatment recommendations. Only 1 low-performing clinic leader mentioned this, and it was in relation to their problems with losses of long-term clinicians:

"But I think...that patients that have a really good relationship with their provider seem to want to follow up and don't want to disappoint their doctor." (Clinic F High)

"If my doctor says that I need to have this done, I need to work on getting that done,' and patients really feel like that here." (Clinic O High)

As we identified these themes, a meta-framework for organizing the relations among themes gradually emerged. Instead of viewing the clinic transformation process as a single step from a traditional focus on the doctor-patient relationship to one involving a larger care team and organized care management processes, the top-performing clinics appeared advance to a new stage of proactive care. Figure 1 summarizes comments across all clinics. Top clinics needed wellfunctioning care management processes, along with few barriers and the right mix of facilitators, but they were also functioning in a third stage of care that depended on adding data-informed outreach and opportunistic proactivity.

\section{DISCUSSION}

Analysis of the content of interviews with leaders of primary care clinics allowed us to understand and characterize strategies, barriers, and facilitators for diabetes care associated with the highest performance measure scores. Although preliminary, what appeared to make the greatest difference in outcome scores was the extent to which clinics had good care management processes for patient visits and also actively and consistently used data to identify needs and proactive methods to engage patients in care. When these strategies were used by clinics that also had fewer barriers and more facilitators, they achieved the best results.

Our quantitative data regarding the clinics and interview findings facilitated the qualitative findings and lessons. By adjusting for socioeconomic factors, we identified clinics for interview that were in upper or lower performance ranks and also performing better or worse than expected. This was confirmed by the lack of a clear relation between performance and the proportion of patients on medical assistance among the interviewed clinics.

Our measure of the presence of systematic care management processes helped confirm our qualitative theme that clinics can obtain good scores by emphasizing these processes, but to achieve the highest levels of performance, it is important to also focus on proactive care. Thus, the middle-performing clinics had greater numbers of these processes than the high- or lowperforming clinics.

When we began our analysis, we thought that highperforming clinics would mostly ascribe their success to the implementation and use of care management processes such as reminder systems, checklists, data audit and feedback, and patient education. Much of the literature on care improvement has been devoted to testing such systems and addressing implementation issues. These systems are reflected in models for care improvement such as the Chronic Care Model and the
Figure 1. The stages of diabetes care strategies.

\begin{tabular}{|c|c|c|}
\hline \multirow{17}{*}{$\begin{array}{c}\text { Barriers } \\
\text { Finances-clinic } \\
\text { and patient } \\
\text { Lack of facilitators } \\
\text { Dysfunction } \\
\text { Other priorities: } \\
\text { Provider/patient } \\
\text { Time } \\
\text { Language } \\
\text { Social determinants } \\
\text { Connection to } \\
\text { resources }\end{array}$} & \multirow{6}{*}{$\begin{array}{l}\text { Stage III-Proactivity } \\
\text { Care teams are further expanded } \\
\text { and use systematically collected } \\
\text { data to reach out proactively and } \\
\text { opportunistically to patients to } \\
\text { encourage care patients did not } \\
\text { know they needed. }\end{array}$} & \multirow{17}{*}{$\begin{array}{c}\text { Facilitators } \\
\text { Access } \\
\text { Continuity } \\
\text { Trust } \\
\text { Relationships } \\
\text { Leadership } \\
\text { Performance awareness } \\
\text { Vision } \\
\text { Culture } \\
\text { Team and patient } \\
\text { engagement } \\
\text { Delegation } \\
\text { Cooperation } \\
\text { Data access and usability } \\
\text { Resources } \\
\text { Usable health informa- } \\
\text { tion technology } \\
\text { Communication }\end{array}$} \\
\hline & & \\
\hline & & \\
\hline & & \\
\hline & & \\
\hline & & \\
\hline & & \\
\hline & Stage II-Systems & \\
\hline & Physicians work together in groups & \\
\hline & $\begin{array}{l}\text { With expanded care teams and sys- } \\
\text { tematic care processes that are still }\end{array}$ & \\
\hline & $\begin{array}{l}\text { largely based on individual patient } \\
\text { complaints and are built around }\end{array}$ & \\
\hline & encounters. & \\
\hline & & \\
\hline & Stage I-Individuality & \\
\hline & $\begin{array}{l}\text { Individual physicians help individ- } \\
\text { ual patients understand and deal }\end{array}$ & \\
\hline & with the health problems patients & \\
\hline & bring, often helped by an assistant. & \\
\hline
\end{tabular}


Bodenheimer Building Blocks. ${ }^{16-23}$ Those models suggest that what distinguishes a transformed medical practice from the traditional model of independent physicians and clinical assistants is largely more consistent and systematically delivered care. We found that the highperforming clinics were indeed using such across-clinic systems, but they were also taking the next step toward proactive, opportunistic, and patient-centered care.

\section{Limitations}

Our findings are based on data and interviews with a relatively small group of clinics in a single state, and they include few low-performing clinics and mostly indirect measures of socioeconomic status (Medicaid status was direct); therefore, they are preliminary and in need of confirmation in other settings. The thematic lessons we developed also likely represent a difference in degree rather than sharply defined differences between clinics. Thus, our data analysis and conceptualization of what we heard should be considered primarily hypothesis generating.

Nevertheless, we believe that our findings are an important extension of the literature on care improvement for chronic conditions. Given that even high-performing clinics were achieving best clinical outcomes for $50 \%$ to $60 \%$ of their patients, there is still more to elucidate regarding improvement of care. Perhaps there are other stages in the development of innovations for practice transformation, in the meantime, these findings should be considered by leaders interested in improving care for patients with diabetes and other chronic conditions.

To read or post commentaries in response to this article, sgo to https://www.AnnFamMed.org/content/19/2/110/tab-e-letters.

Submitted March 16, 2020; submitted, revised, June 2, 2020; accepted June 22, 2020.

Key words: quality of health care; diabetes mellitus; delivery of health care; primary health care; organizational culture

Funding support: This research was supported by the National Institute of Diabetes and Digestive and Kidney Diseases of the National Institutes of Health (\#R18DK110732). The content is solely the responsibility of the authors and does not necessarily represent the official views of the National Institutes of Health.

Reporting guidelines: COREQ Checklist

Acknowledgments: We are grateful to those who made this analysis and paper possible, especially to the leaders of the 17 clinics who took the time to thoughtfully respond to our interview questions. Without the standardized performance measure data from MN Community Measurement, we would have had no way to identify the relative performance among clinics, limiting our findings to the usual qualitative study of opinions without relation to success.
2. Centers for Disease Control and Prevention (CDC). Million hearts: strategies to reduce the prevalence of leading cardiovascular disease risk factors--United States, 2011. MMWR Morb Mortal W/kly Rep. 2011;60(36):1248-1251.

3. Siminerio LM, Albright A, Fradkin J, et al. The National Diabetes Education Program at 20 years: lessons learned and plans for the future. Diabetes Care. 2018;41(2):209-218.

4. Shojania KG, Ranji SR, McDonald KM, et al. Effects of quality improvement strategies for type 2 diabetes on glycemic control: a meta-regression analysis. JAMA. 2006;296(4):427-440.

5. Tummalapalli SL, Powe NR, Keyhani S. Trends in quality of care for patients with CKD in the United States. Clin J Am Soc Nephrol. 2019; 14(8):1142-1150.

6. McCullough JS, Crespin DJ, Abraham JM, Christianson JB, Finch M. Public reporting and the evolution of diabetes quality. Int J Health Econ Manag. 2015;15(1):127-138.

7. Peterson KA, Carlin C, Solberg LI, Jacobsen R, Kriel T, Eder M. Redesigning primary care to improve diabetes outcomes (the UNITED study). Diabetes Care. 2020;43(3):549-555.

8. Solberg LI, Carlin C, Peterson KA, Eder M. Differences in diabetes care with and without certification as a medical home. Ann Fam Med. 2020;18(1):66-72.

9. Scholle SH, Pawlson LG, Solberg LI, et al. Measuring practice systems for chronic illness care: accuracy of self-reports from clinical personnel. Jt Comm J Qual Patient Saf. 2008;34(7):407-416.

10. Solberg LI, Asche SE, Pawlson LG, Scholle SH, Shih SC. Practice systems are associated with high-quality care for diabetes. Am J Manag Care. 2008;14(2):85-92.

11. Solberg LI, Crain AL, Jaeckels N, et al. The DIAMOND initiative: implementing collaborative care for depression in 75 primary care clinics. Implement Sci. 2013;8:135.

12. United States Census Bureau. American Community Survey. Accessed Sep 23, 2018. https://www.census.gov/programs-surveys/acs

13. Hsieh HF, Shannon SE. Three approaches to qualitative content analysis. Qual Health Res. 2005;15(9):1277-1288.

14. Saldaña J. The Coding Manual for Qualitative Researchers. Second edition. Sage Publications; 2013.

15. Padgett DK. Qualitative Methods in Social Work Research. Third edition. Sage Publications; 2017.

16. Wagner EH, Austin BT, Davis C, Hindmarsh M, Schaefer J, Bonomi A. Improving chronic illness care: translating evidence into action. Health Aff (Millwood). 2001;20(6):64-78.

17. Bodenheimer T, Wagner EH, Grumbach K. Improving primary care for patients with chronic illness: the chronic care model, part 2. JAMA. 2002;288(15):1909-1914.

18. Bodenheimer $\mathrm{T}$, Wagner $\mathrm{EH}$, Grumbach $\mathrm{K}$. Improving primary care for patients with chronic illness. JAMA. 2002;288(14):1775-1779.

19. Solberg LI, Crain AL, Sperl-Hillen JM, Hroscikoski MC, Engebretson $\mathrm{KI}, \mathrm{O}$ 'Connor PJ. Care quality and implementation of the chronic care model: a quantitative study. Ann Fam Med. 2006;4(4):310-316.

20. Halladay JR, DeWalt DA, Wise A, et al. More extensive implementation of the chronic care model is associated with better lipid control in diabetes. J Am Board Fam Med. 2014;27(1):34-41.

21. Berwick DM. Reflections on the chronic care model-23 years later. Milbank Q. 2019;97(3):665-668.

22. Bodenheimer T, Ghorob A, Willard-Grace R, Grumbach K. The 10 building blocks of high-performing primary care. Ann Fam Med. 2014;12(2):166-171.

23. Nelson EC, Batalden PB, Huber TP, et al. Microsystems in health care: part 1. Learning from high-performing front-line clinical units. Jt Comm J Qual Improv. 2002;28(9):472-493.

\section{References}

1. Kazemian P, Shebl FM, McCann N, Walensky RP, Wexler DJ. Evaluation of the cascade of diabetes care in the United States, 20052016. JAMA Intern Med. 2019;179(10):1376-1385. 